\title{
Los modelos de prácticas profesionales en España y Noruega: una comparativa de las rutinas y condiciones de los estudiantes de Periodismo
}

\author{
Internship models in Spain and Norway: \\ comparative of Journalism students' media \\ routines and working conditions
}

\section{RESUMEN:}

Este artículo plantea una comparativa entre las prácticas profesionales de los estudiantes de Periodismo de España y los de Noruega. Para ello se realizó una primera encuesta en octubre de 2018 en la Universidad de Málaga y una segunda en junio de 2019 en las de Stavanger y Bergen $(n=46)$. El cuestionario, idéntico para ambos grupos, preguntaba sobre las rutinas, condiciones y satisfacción de los estudiantes durante sus prácticas curriculares (en el caso de Noruega) y extracurriculares (en el caso de Málaga), al no existir estancias plenamente homologables. En Noruega los estudiantes reciben unos emolumentos muy superiores en promedio a los de España (1.145 euros frente a 384$)$ por unas jornadas más prolongadas en el tiempo $(8,07$ horas frente a 6,63$)$ pero menos intensas en cuanto a producción informativa $(1,17$ piezas diarias frente a 4,62). Los resultados evidencian la existencia de dos modelos con diferencias destacables: en Noruega también es más tenido en cuenta el criterio de los estudiantes, y el contacto con los tutores responsables de su estancia es mucho más fluido. En consecuencia, los niveles de satisfacción son netamente superiores. Este artículo constituye la primera comparativa de las prácticas en España con las de otro país y contribuye a la reflexión sobre la necesaria reforma del sistema de prácticas nacional.

\section{PALABRAS CLAVE:}

Prácticas profesionales; Periodismo; Rutinas profesionales; Condiciones laborales; Universidad; Formación.

\section{ABSTRACT:}

This article compares the media internships of Journalism students in Spain and Norway. For this, a first survey was carried out in October 2018 at the University of Malaga and a second one in June 2019 at the universities of Stavanger and Bergen $(n=46)$. The questionnaire, identical for both groups, asked about their routines, conditions and satisfaction during their curricular (in the case of Norway) and extracurricular internships (in the case of Malaga) - since there were no fully comparable stays. Norwegian students receive a much higher income than the Spanish ones (an average of 
1,145 euros versus 384 ) for longer workdays (8.07 hours versus 6.63), although less intense in terms of production (1.17 daily pieces versus 4.62). The results show the existence of two models with notable differences: in Norway, the students' opinions are taken into account more frequently, and the contact with the tutors responsible for their internships is much more fluid. Consequently, the levels of satisfaction are clearly higher. This article constitutes the first comparison of Spain internships with those of another country, and contributes to the reflection on the necessary reform of the national internship system.

KEY WORDS:

Media internships; Journalism; Media routines; Working conditions; University; Training.

\section{Introducción y objetivos}

Existe un gran consenso en la comunidad académica en torno al amplio margen de mejora del que disponen las universidades a la hora de abordar la regulación de las prácticas profesionales de los estudiantes, en particular en el ámbito del Periodismo. Desde los primeros trabajos de Cantalapiedra, Coca y Bezunartea (2000) hasta los más recientes (Gómez-Calderón, García-Borrego y Fernández-Sande, 2019) se ha descrito una realidad de precariedad que cuenta con la venia de la propia universidad, con la cual se contribuye, además, a perpetuar el inestable escenario en el que también viven los profesionales consolidados, ya que en buena medida los estudiantes acaban sustituyendo a redactores de plantilla por unos costes mínimos para la empresa (Blanco, 2005; Lamuedra, 2007; García-Borrego, Roses y Farias, 2017). Pese a encontrarse los estudiantes en una fase crucial en la configuración de sus ideas sobre la profesión, de la que dependerá en gran parte su futuro laboral (Ashforth, Sluss y Harrison, 2007; Cotter, 2010; Mellado et al., 2013; Gravengaard y Rimestad, 2014), los estudios sobre prácticas en empresas son contados, y tienden a mostrar una mejora paulatina, aunque lenta, de las condiciones que caracterizan a estas estancias formativas.

En el País Vasco, donde se iniciaron los estudios sobre prácticas profesionales, se hablaba de cómo «la figura de prácticas-becario», que se contaba por centenares, había reemplazado a la del periodista sénior en un número considerable de medios, muchos de ellos dependientes de los propios estudiantes, sin los cuales no podrían subsistir, y que por lo general no recibían ningún tipo de compensación económica por sus jornadas a tiempo completo (Cantalapiedra, Coca y Bezunartea, 2000).

En Málaga, años más tarde, se describía un panorama algo más amable: al menos dos tercios de los estudiantes eran remunerados por sus tareas - aunque en mayor medida los hombres que las mujeres - y por lo general la cuantía oscilaba entre los 200 y los 300 euros (Blanco, 2005). Aun así, el exceso de horas y la percepción de que los medios abusaban de este tipo de figuras seguían siendo la norma, si bien desde entonces las condiciones de las prácticas han experimentado una mejora tangible: los últimos estudios recogen que los horarios se sitúan por debajo de las siete horas y la retribución se acerca lentamente a los 400 euros de promedio (García-Borrego, Roses y Farias, 2017; Gómez-Calderón, GarcíaBorrego y Fernández-Sande, 2019).

En Madrid, el otro foco desde donde se han llevado a cabo estudios de este tipo, los estudiantes de la Universidad Complutense perciben una ayuda media de 268,0 euros, lejos de los 375,7 de Málaga pero ligeramente por encima de los 223,3 que se detectaban en el período 2012-2014 (Pérez-Serrano, Rodríguez-Barba y Rodríguez-Pallares, 2015; Gómez-Calderón, 
García-Borrego y Fernández-Sande, 2019). En compensación, dedican unos 20 minutos menos al día que los de Málaga, en torno a seis horas, aunque superan las cinco que de promedio se marcan en las ofertas de prácticas.

El estudio a escala nacional de las normativas que rigen las prácticas extracurriculares en el grado en Periodismo no invita a pensar que el fenómeno descrito se circunscriba en exclusiva a los tres territorios señalados, sino más bien al contrario, a la vista de que la mitad de las universidades públicas otorgan total libertad a las entidades colaboradoras para establecer la duración de las jornadas de prácticas y tres cuartas partes hacen lo propio con la ayuda a recibir por los alumnos (García-Borrego, Gómez-Calderón y Farias, 2020). Solo tres centros, de hecho, fijan topes horarios y mínimos de remuneración: Universidad Carlos III de Madrid, la propia Universidad de Málaga y la Universidad de Sevilla.

Es en este contexto, en el que la finalidad formativa de las prácticas profesionales en España parece haber sido desplazada por las necesidades productivas de los medios, donde se plantea la realización de este trabajo, el primero de tipo comparativo en este campo de conocimiento. Con él se persiguen fundamentalmente dos objetivos:

- O1. Describir y comparar las prácticas en empresas en España con las de un modelo de referencia, como el de Noruega, atendiendo a elementos diferenciadores como las condiciones en que estas tienen lugar, las rutinas profesionales seguidas o los índices de satisfacción de los estudiantes.

- O2. Definir las características de los modelos de cada uno de los países seleccionados y favorecer así el debate para implementar mejoras en el sistema de prácticas español.

\subsection{Marco de las prácticas en España y Noruega}

En España las prácticas externas se rigen por el Real Decreto 592/2014, de 11 de julio, que distingue entre las curriculares -integradas en el plan de estudios y por lo general no remuneradas - y las extracurriculares - de carácter voluntario y complementario, frecuentemente remuneradas - . Ambas «deberán ofrecerse preferentemente en la segunda mitad del plan de estudios» (véase el artículo 12.6 del Real Decreto 1393/2007, de 29 de octubre) y «tendrán una duración preferentemente no superior al cincuenta por ciento del curso académico, sin perjuicio de lo que fijen las universidades" (artículo 5.1.b del RD 592/2014), también encargadas de regular, si así lo estiman conveniente, otros aspectos como los horarios y la remuneración. En concreto, la Universidad de Málaga indica que «se tendrá como preferencia una duración no superior a cinco horas diarias de prácticas o un cómputo global de 100 horas al mes» (Universidad de Málaga, 2016) y que las extracurriculares concretamente «conllevan una contraprestación económica mínima de $360 €$ y alta en Seguridad Social»1.

En este sentido, el modelo dista bastante de otros como el noruego, donde no existen las prácticas extracurriculares, y las curriculares, remuneradas, se incluyen de manera obligatoria como parte de los planes de estudios. En la Universidad de Bergen consisten en dos

1/ Guía de prácticas académicas externas de la UMA: https://www.uma.es/media/files/Guia_de_practicas_externas_UMA.pdf 
períodos de ocho semanas cada uno a lo largo del segundo año de los tres que componen el programa ${ }^{2}$; en Stavanger, las estancias son de diez semanas cada una ${ }^{3}$, y algo similar ocurre en Oslo $^{4}$ y Volda ${ }^{5}$. Todas ellas se encuentran entre las principales universidades noruegas con estudios superiores de Periodismo. Estas prácticas suelen estar remuneradas con el $40 \%$ del salario mínimo, y a menudo derivan en un contrato laboral para la temporada de verano, aspecto en el que guarda ciertas semejanzas con España, donde las estancias curriculares conducen en ocasiones a contratos de prácticas extracurriculares en el futuro.

No se han localizado estudios que aborden específicamente las condiciones y rutinas de las prácticas en Noruega -más allá de algunas investigaciones como la de Bjørnsen, Hovden y Ottosen (2007), que muestran un declive palpable en los ideales profesionales de los estudiantes que las han realizado-, pero sirven como muestra los trabajos de países análogos como Dinamarca, donde los alumnos perciben durante sus estudios en torno a 2.400 euros mensuales por jornadas casi «idénticas» a las de un profesional consolidado (Gravengaard y Rimestad, 2014, p. 81).

\section{Metodología}

El trabajo de campo de este artículo de basa en dos encuestas a estudiantes de Periodismo. La primera se llevó a cabo en la Universidad de Málaga, España, a lo largo del mes de octubre de 2018, con un primer envío el día 15 y un segundo el 22 a las tres últimas promociones del grado en Periodismo $(n=345)$, dado que por motivos de protección de datos no fue posible identificar previamente a los alumnos que habían realizado prácticas $(89$, según los datos facilitados por el Servicio de Empleabilidad y Emprendimiento de la universidad). En el mensaje se pidió la respuesta únicamente de aquellos que hubieran disfrutado de este período de formación dentro del curso inmediatamente anterior (2017-2018) para evitar distorsiones en el recuerdo de la experiencia. Al término del mes se obtuvo un total de 59 respuestas, representativas del $66,3 \%$ de la población. De este cómputo se excluyó a posteriori a los 20 estudiantes que habían recalado en gabinetes de prensa, manteniendo únicamente a los que ejercieron tareas puramente periodísticas para permitir así la comparativa con el contexto noruego, dado que los alumnos de este tipo de carreras no suelen desempeñar tareas de comunicación institucional y corporativa. La muestra final de España, por tanto, asciende a 39 alumnos.

La encuesta en Noruega se puso en marcha en la primera quincena de junio de 2019. Para suministrar por vía telemática el cuestionario se trabó contacto con el profesorado responsable de cada centro para pedir que remitieran el enlace a los estudiantes de segundo año. Inicialmente se planteó incluir solo a la Universidad de Bergen $(n=22)$ en la muestra, pero el

\footnotetext{
2/ Journalistisk praksis, Journalistikk, bachelor, 3 år. Universitetet i Bergen: https://www.uib.no/emne/ JOU201

3/ Journalistisk praksis, Bachelor i journalistikk. Universitetet i Stavanger: https://www.uis.no/course/ ?code=BJOPRA_1\&parentcat=9086

4/ Praksis for journalistikk, Journalistikk Bachelorprogram. Oslomet: https://student.oslomet.no/ praksis-journalistikk

5/ Studieplan, Journalistikk, spesialisering tv/radio. Høgskulen i Volda: https://www.hivolda.no/studietilbod/ journalistikk-spesialisering-tvradio/haust
} 
bajo número de respuestas alcanzado motivó la ampliación a la Universidad de Stavanger, con características similares $(n=25)$. En última instancia se recibieron siete contestaciones al cuestionario, un número modesto (14,9\% de la población) que, sin embargo, permite extraer resultados de interés debido a la uniformidad de las respuestas proporcionadas.

La selección de los centros, tanto en Noruega como en España, se explica por razones de conveniencia de los investigadores participantes y por similitud en las características de los centros: universidades de la periferia con un número de estudiantes más modesto que en las respectivas capitales. Se procuró, pues, favorecer en todo momento el trabajo comparativo.

El cuestionario se elaboró de acuerdo con los principales estudios de referencia sobre prácticas en empresas y profesión periodística (entre otros: APM, 2005-2019; Blanco, 2005; Lamuedra, 2007; Weaver y Willnat, 2012; Túñez-López y Martínez-Solana, 2014; SuárezVillegas, 2015; García-Borrego, Roses y Farias, 2017), y se estructuraba en torno a cuatro bloques: un primero con información de registro básica sobre el estudiante, un segundo sobre las condiciones en que se desarrollan las prácticas, un tercero sobre rutinas profesionales y un cuarto sobre niveles de satisfacción y expectativas futuras. La única diferencia entre los formularios enviados a uno y otro colectivo radica en las preguntas sobre la temporada en que transcurrieron las prácticas: mientras que en España se cuestionaba sobre si tuvieron lugar durante el verano o en período lectivo, en Noruega, al tratarse de un marco temporal prefijado por la universidad, se preguntaba si las prácticas habían ayudado a conseguir un contrato laboral en época estival -y, en tal caso, cuál había sido la remuneración.

\subsection{Descripción de LA MUestra}

Existen algunas diferencias apreciables en la composición de cada una de las muestras. En primer lugar, en la proporción de hombres, superior entre los estudiantes noruegos pese a que, como en España, suelen ocupar entre el $30 \%$ y el $40 \%$ de las plazas de la titulación de Periodismo. También existe disparidad en el año de matriculación de los alumnos por las características del sistema: mientras que en España la mayoría (84,6\%) pertenece al menos a cuarto curso, cuando no han terminado el grado o apenas se matricularon de unos créditos pendientes, en la muestra noruega son en su totalidad estudiantes segundo curso. A pesar de ello, la edad media de ambos grupos es similar, superando por poco los 23 años. El resto de los datos relevantes se pueden consultar en la tabla 1

Tabla 1. Composición de la muestra.

\begin{tabular}{|c|c|c|c|c|}
\hline & \multicolumn{2}{|c|}{ España } & \multicolumn{2}{|c|}{ Noruega } \\
\hline $\mathrm{N}$ & \multicolumn{2}{|c|}{39} & \multicolumn{2}{|c|}{7} \\
\hline Género & Hombre $=46,20 \%$ & Mujer $=53,80 \%$ & Hombre $=71,40 \%$ & Mujer = 28,60\% \\
\hline Edad & Media $=23,03$ & $\mathrm{DE}=2,22$ & Media $=23,14$ & $\mathrm{DE}=1,68$ \\
\hline Universidad & $U M A=100,0 \%$ & & Bergen $=28,6 \%$ & Stavanger $=71,4 \%$ \\
\hline Curso & $3^{\circ}=15,4 \%$ & $4^{\circ}$ o sup. $=84,6 \%$ & $2^{\circ}=100,0 \%$ & \\
\hline
\end{tabular}


Tabla 2. Horarios y piezas realizadas durante las prácticas

\begin{tabular}{|l|c|c|}
\hline & España & Noruega \\
\hline Promedio de horas diarias & 6,63 & 8,07 \\
\hline Tienen turno partido & $46,2 \%$ & $42,9 \%$ \\
\hline Promedio de piezas diarias & 4,62 & 1,17 \\
\hline
\end{tabular}

\section{Resultados}

\subsection{Condiciones de LAS PRÁcticas}

La duración de una jornada de prácticas en Noruega equivale a la de un contrato laboral estándar, superando por escasos minutos las 8 horas de media. El estudiante con menor dedicación se sitúa en 7 diarias; el que más, en 9,5. En Málaga, en cambio, la duración suele ser inferior (6,63 horas de promedio), aunque supera la cifra de 5 horas diarias que recomienda el centro. No obstante, un $34,2 \%$ de la muestra española iguala o supera las jornadas de 8 horas, y existen varios casos que afirman alcanzar las 10 y las 11 horas diarias.

Sin embargo, en Noruega las rutinas de producción son mucho más relajadas, y los estudiantes realizan apenas 1,17 piezas diarias frente al promedio cuatro veces superior de Málaga $(4,62)$. De hecho, todos los estudiantes noruegos elaboran tan solo una pieza diaria, con la excepción de uno que elabora dos y otro que no contesta a la pregunta - quizá por no realizar ninguna-, mientras que en Málaga el 97,4\% confecciona dos o más piezas, con máximos de 10, 11, 12 y hasta 15 (Tabla 2).

En lo relativo a la remuneración, la de los estudiantes noruegos es claramente superior a la de los españoles, posiblemente más de lo que puede preverse dados los niveles de vida de cada país. En Málaga, la ayuda media que reciben los alumnos que realizan sus prácticas en redacciones es de 384,20 euros, es decir, supera en casi 25 euros el mínimo de 360 establecido por el centro, en este aspecto uno de los más garantistas de España. En Noruega, pese a tratarse de prácticas curriculares incluidas en el plan de estudios, la remuneración triplica a la de Málaga con 1.145,84 euros de promedio ${ }^{6}$. Los tres alumnos peor retribuidos perciben 986,88 euros (10.000 coronas noruegas en la respuesta original); el que más, 1.408,78.

Para cinco de los siete estudiantes noruegos encuestados estas prácticas curriculares se materializaron en un contrato de trabajo durante los meses de verano, con la consiguiente mejora en las condiciones económicas. El salario medio de estas estancias para estudiantes de segundo curso fue de $3.213,53$ euros, con un mínimo de 2.664,59 euros y un máximo de 3.521,89 euros (Gráfico 1).

6/ La conversión se realizó de acuerdo con los datos de referencia de Morningstar del 16 de octubre de 2019 a las 11.32 horas de la mañana, cuando 1 euro equivalía a 10,13 coronas noruegas. Esta cifra se ha mantenido estable en los últimos años salvo en los momentos más agudos de la crisis del coronavirus ( $1 €=12,66 \mathrm{NOK}$ el 20 de marzo de 2020). Actualmente la conversión se aproxima poco a poco a los niveles anteriores ( $1 €=10,86$ NOK el 26 de junio de 2020 a las 11.17 de la mañana). 


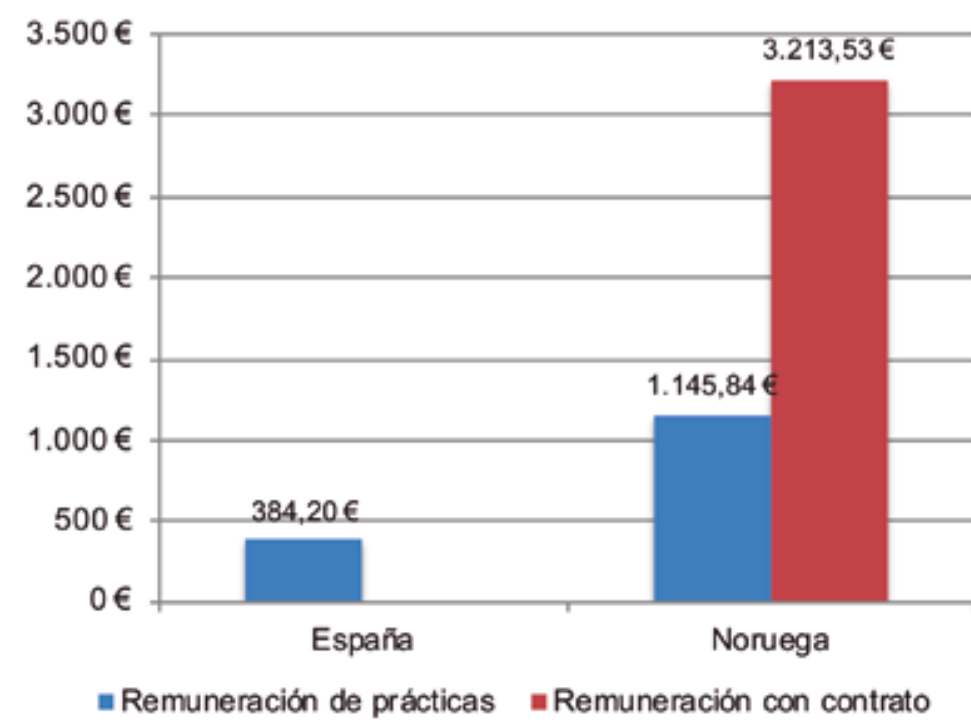

Gráfico 1. Remuneración de las prácticas.

\subsection{Rutinas profesionales}

En relación con las rutinas, los estudiantes suelen proponer temas en una medida similar independientemente del país de referencia: el 34,2\% de los españoles lo hace siempre por el $28,6 \%$ de los noruegos, y el $18,4 \%$ de los primeros lo hace solo rara vez por el $14,3 \%$ de los segundos. En cambio, en el resto de variables estudiadas, aspectos como la intervención en el enfoque, la firma de textos con los que se encuentran disconformes, la realización de tareas contrarias a sus principios o el contacto con los tutores, las divergencias son más notables.

A pesar de que la iniciativa es pareja en la propuesta de temas, el modelo de prácticas noruego parece dotar a sus estudiantes de mayor libertad a la hora de escoger el enfoque de las informaciones: el $71,4 \%$ lo decide siempre, o al menos ve escuchada su opinión, una cifra que solo alcanza el $15,4 \%$ en España. Estos datos casan con que en España resulte menos común que los estudiantes de Periodismo firmen piezas con las que no se encuentren cómodos por los cambios sufridos con respecto al original. El 42,9\% no lo ha hecho nunca (35,9\% en Málaga) y el resto afirma haberlo hecho rara vez; por tanto, ninguno asegura sufrir este fenómeno habitualmente o siempre, mientras que en Málaga este colectivo representa un $20,5 \%$ del total.

Cuando se lleva esta consulta al terreno ético o profesional, la brecha es aún mayor: en Noruega solo un estudiante ha llevado a cabo tareas con las que no está de acuerdo por ir en contra de sus principios, aunque esto solo ha sucedido rara vez. El 85,7\% restante, por tanto, no se ha visto en esta coyuntura. En España lo ha hecho alguna vez el 53,8\% de la muestra, y de ellos algo menos de la mitad se enfrenta a esta contradicción de manera asidua o diaria, como se observa en el gráfico 2.

Parte de la responsabilidad por estas cifras recae necesariamente en los tutores. En España es mucho menos fluido el contacto entre los alumnos y los encargados de velar por 


\section{Ha realizado tareas contrarias a la ética}

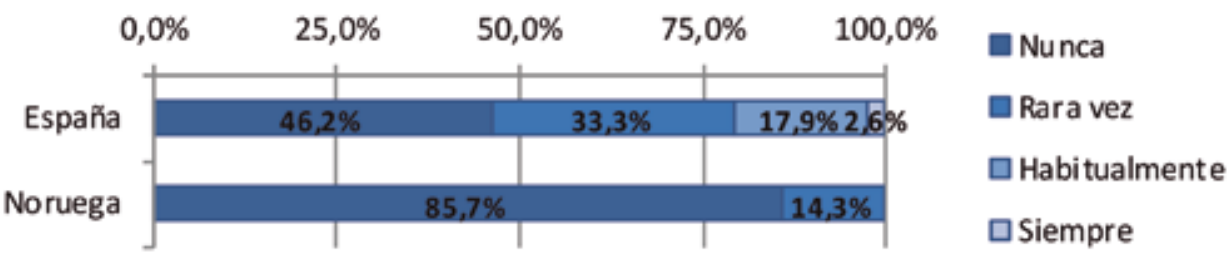

Gráfico 2. Frecuencia con la que ha realizado tareas con las que no está de acuerdo por razones éticas o profesionales.

el adecuado cumplimiento de las prácticas, hasta el punto de que el 43,6\% nunca ha contactado con su tutor de empresa ( $0 \%$ de casos en Noruega) y el $94,9 \%$ no ha establecido relaciones con el de la universidad (28,6\% en Noruega). En ambos países el responsable de la institución de acogida es la principal referencia para el estudiante, si bien en Noruega la ratio de estudiantes que acude a su tutor profesional más de una vez por semana $(85,7 \%)$ duplica al español (41,0\%) (Tabla 3).

Tabla 3. Frecuencia de contacto con los tutores profesional y académico.

\begin{tabular}{|l|c|c|c|c|}
\hline & \multicolumn{2}{|c|}{ España } & \multicolumn{2}{c|}{ Noruega } \\
\cline { 2 - 5 } & Nunca & $\geq 1 /$ semana & Nunca & $\geq 1$ / semana \\
\hline Tutor de empresa & $43,6 \%$ & $41,0 \%$ & $0,0 \%$ & $85,7 \%$ \\
\hline Tutor de universidad & $94,9 \%$ & $0,0 \%$ & $28,6 \%$ & $0,0 \%$ \\
\hline
\end{tabular}

\subsection{Niveles de SATisfacción}

En último lugar, en lo referente a los niveles de satisfacción, las diferencias vuelven a resultar más que evidentes entre ambos modelos de prácticas. De los 11 indicadores de satisfacción profesional que se contemplan en este trabajo, los estudiantes noruegos puntúan mejor en 10. Los españoles solo se encontrarían más cómodos con el resto de los trabajadores del medio, y arrojan puntuaciones muy inferiores en las variables relacionadas con la labor del tutor académico, los horarios, la remuneración, la estabilidad de su relación con la empresa, las oportunidades de promocionar o la línea editorial del medio. Los promedios de cada ítem -en escala de 1 a 5 - se recogen en la tabla 4.

Esta distancia en los índices de satisfacción se refleja igualmente en las aspiraciones de los estudiantes en el plazo de cinco años. Casi la mitad de los noruegos (42,9\%) preferirían permanecer en su medio, por solo un $12,8 \%$ de los españoles, que mayoritariamente cambiarían de medio. Curiosamente, el desencanto en Noruega parece relacionarse más con la opción laboral de la comunicación corporativa, mientras que en Málaga esta opción gozaría de mucho menor predicamento (Tabla 5). 
Tabla 4. Satisfacción con las prácticas por ítem

\begin{tabular}{|l|c|c|}
\hline & España & Noruega \\
\hline Tutor de empresa & 2,90 & 3,00 \\
\hline Tutor académico & 1,56 & 3,14 \\
\hline Trabajadores de la empresa & 3,87 & 3,57 \\
\hline Otros estudiantes & 4,00 & 4,29 \\
\hline Superiores & 3,13 & 3,86 \\
\hline Horarios & 2,74 & 3,57 \\
\hline Estabilidad de las prácticas & 2,41 & 4,00 \\
\hline Carga de trabajo & 2,67 & 3,29 \\
\hline Línea editorial & 2,73 & 3,43 \\
\hline Oportunidades de promocionar & 2,21 & 3,71 \\
\hline Remuneración & 1,95 & 3,57 \\
\hline Media & 2,58 \\
\hline
\end{tabular}

Tabla 5. Aspiraciones de los estudiantes en el plazo de cinco años.

\begin{tabular}{|l|c|c|}
\hline & España & Noruega \\
\hline Querría permanecer en su medio & $12,8 \%$ & $42,9 \%$ \\
\hline Querría trabajar en otro medio & $51,3 \%$ & $14,3 \%$ \\
\hline Querría trabajar en un gabinete & $2,6 \%$ & $28,6 \%$ \\
\hline No lo tiene claro & $25,6 \%$ & $14,3 \%$ \\
\hline $\begin{array}{l}\text { Querría dejar el campo de la } \\
\text { comunicación }\end{array}$ & $7,7 \%$ & $0,0 \%$ \\
\hline
\end{tabular}

\section{Conclusiones/Discusión}

Este trabajo ha analizado someramente las principales similitudes en los programas de prácticas externas en España y Noruega a partir de los ejemplos de Málaga, Bergen y Stavanger. Se trata del primer trabajo que compara este tipo de estancias formativas en España con las de otro país, y aunque algunas de las variables pueden verse condicionadas por el tamaño de la muestra, en líneas generales los datos ofrecen evidencias suficientes para responder a los objetivos planteados. 
Como 01 se fijaba la descripción y comparación de los aspectos que dan forma a las prácticas en España y Noruega. Más allá de los aspectos formales, como que los noruegos las llevan a cabo durante el segundo curso, donde estas operan como una asignatura obligatoria, y que los españoles las escojan libremente a partir del tercer año como complemento opcional a su formación en las aulas, se dan diferencias claras en el desarrollo de estas estancias.

En primer lugar, Noruega destaca, claramente, por la alta remuneración de sus estudiantes, que durante el curso triplica el promedio de los alumnos de Málaga y en período estival -ya con un contrato temporal - lo octuplica, pese a que el coste de vida en el país nórdico supone algo menos del doble del español. Se trata de estudiantes en el ecuador de su formación periodística que cursan una asignatura obligatoria (prácticas curriculares), mientras que en España abundan los estudiantes de cuarto curso, e incluso aquellos que han prorrogado voluntariamente sus estudios para seguir aspirando a realizar estas prácticas voluntarias (extracurriculares). Se da, además, la circunstancia adicional de que Málaga es una de las universidades más garantistas en lo referido a la ayuda recibida por los estudiantes: la gran mayoría de los centros españoles no estipulan retribuciones mínimas para las prácticas, lo que da lugar a que en universidades como la Complutense de Madrid la remuneración media sea considerablemente inferior (Gómez-Calderón, García-Borrego y Fernández-Sande, 2019; García-Borrego, Gómez-Calderón y Farias, 2020).

Parte de esta diferencia puede explicarse por el tipo de jornada, que mientras que en España tiende a asociarse con una duración más reducida, en Noruega equivale a la de un periodista sénior. No obstante, el número de piezas realizadas al día es muy inferior, lo cual apunta principalmente a dos posibilidades: bien los alumnos noruegos dedican buena parte del tiempo al propio aprendizaje y adquisición de los mecanismos y rutinas básicos para su formación como periodistas, quizá desde una perspectiva menos activa que en el contexto español, bien acostumbran a un periodismo de mayor profundidad y, por ello, dedican la mayor parte de su tiempo a trabajar en una misma historia. El ítem que mide la satisfacción con la carga de trabajo no es lo suficiente clarificador al respecto: la discreta conformidad expresada por los estudiantes noruegos puede deberse tanto a que preferirían una cobertura de temas más amplia y dinámica como a justo lo contrario. Futuros estudios podrán abundar en este aspecto por resolver.

Igualmente resultan ilustrativas las divergencias encontradas en el resto de rutinas: la universidad noruega parece profesar un mayor cuidado al estudiante al llevar a cabo una supervisión más generosa de su actividad, lo que parece traducirse en una mayor libertad a la hora de orientar las piezas y en un contacto menos frecuente con el lado amargo de la profesión, manifestado en la realización tareas poco profesionales, o incluso contrarias a la ética de los alumnos, o en la firma de piezas con las que no se sienten cómodos. Estas variables, en cualquier caso, son las más sensibles al reducido tamaño de la muestra, por lo que han de ser tomadas con cautela, aunque apuntan de manera coordinada a una misma realidad.

Con estas precisiones se ha respondido, al mismo tiempo, al segundo objetivo (O2): el modelo noruego parece destacar por un mayor foco en la protección y el aprendizaje del estudiante, mientras que en el español parece primar ante todo la producción. La relativamente alta satisfacción mostrada por los estudiantes nórdicos en indicadores cruciales como el

7/ Datos extraídos del Índice de Coste de Vida por País de 2020 del portal Numbeo. 
papel de los tutores o las condiciones materiales de las prácticas apuntan a la necesidad de una revisión global del sistema de prácticas nacional que tenga en cuenta estos aportes. Bien es cierto que Noruega y España se encuentran en universos distintos tanto por sus distintas realidades laborales como por el estado de la industria mediática; en este sentido, el presente trabajo ofrece pistas sobre cuáles pueden ser algunos de los pasos a dar si se quiere proceder a cerrar esa brecha, que, como se ha visto, no solo depende del elemento pecuniario.

\section{Bibliografía}

APM (2005-2019). Informe anual de la profesión periodística. Madrid: Asociación de la Prensa de Madrid. Recuperado de https://www.apmadrid.es/publicaciones/informe-anual-de-la-profesion/

ASHFORTH, Blake E., SLUSS, David M. y HARRISON, Spencer H. (2007). «Socialization in organizational contexts». International Review of Industrial and Organizational Psychology, 22, 1-70. DOI: 10.1002/9780470753378.ch1

BJØRNSEN, Gunn, HOVDEN, Jan Fredrik y OTTOSEN, Runne (2007). "Journalists in the making: findings from a longitudinal study of Norwegian journalism students». Journalism Practice, 1(3), 383403. DOI: $10.1080 / 17512780701505085$

BLANCO, Elena (2005). «Mujer y poder en los medios: Dificultades para una incorporación plena». En APM. Informe anual de la profesión periodística 2005 (pp. 99-106). Madrid, España: Asociación de la Prensa de Madrid.

CANTALAPIEDRA, María José, COCA, César y BEZUNARTEA, Ofa (2000). «La situación profesional y laboral de los periodistas vascos». Zer - Revista de Estudios de Comunicación, 9, 169-182. Recuperado de http://www.ehu.eus/ojs/index.php/Zer/article/view/17441

COTTER, Colleen (2010). News talk. Investigating the language of journalism. Cambridge, Reino Unido: Cambridge University Press.

GARCÍA-BORREGO, Manuel, ROSES, Sergio y FARIAS, Pedro (2017). "Condiciones de las prácticas profesionales en medios de comunicación: un estudio empírico». Revista Latina de Comunicación Social, 72, 430-452. DOI: 10.4185/RLCS-2017-1173es

GARCÍA-BORREGO, Manuel, GÓMEZ-CALDERÓN, Bernardo y FARIAS, Pedro (2020). «Las normativas de prácticas de las universidades públicas españolas con grado en Periodismo: entre el garantismo y la desregulación». Estudios sobre el mensaje periodístico, 26(3). DOI: 10.5209/ esmp.66588

GÓMEZ-CALDERÓN, Bernardo, GARCíA-BORREGO, Manuel y FERNÁNDEZ-SANDE, Manuel (2019). «Prácticas extracurriculares en el grado en Periodismo: rutinas profesionales, condicionantes y nivel de satisfacción de los alumnos». El Profesional de la Información, 28(6), 1-11. DOI: 10.3145/ epi.2019.nov.10

GRAVENGAARD, Gitte y RIMESTAD, Lene (2014). "Socializing journalist trainees in the newsroom: On how to capture the intangible parts of the process». Nordicom Review, 35, 81-95. Recuperado de https://rimestad.dk/onewebmedia/gravengaard_rimestad.pdf

LAMUEDRA, María (2007). «Estudiantes de Periodismo y prácticas profesionales: el reto del aprendizaje». Comunicar, 28, 203-211. DOI: 10.3916/25995

MELLADO, Claudia, HANUSCH, Folker, HUMANES, María Luisa, ROSES, Sergio, PEREIRA, Fábio, YEZ, Lyuba, DE LEÓN, Salvador, MÁRQUEZ, Mireya, SUBERVI, Federico y WYSS, Vinzenz 
(2013). «The presocialization of future journalists». Journalism Studies, 14(6), 857-874. DOI: 10.1080/1461670X.2012.746006

PÉREZ-SERRANO, María José, RODRíGUEZ-BARBA, Dolores y RODRíGUEZ-PALLARES, Miriam (2015). «Mercado de la comunicación y estudiantes de Periodismo. Estructura de la demanda de perfiles profesionales». Revista Latina de Comunicación Social, 70, 209-229. DOI: 10.4185/ RLCS-2015-1043

REAL DECRETO 1393/2007, de 29 de octubre, por el que se establece la ordenación de las enseñanzas universitarias oficiales. Boletín Oficial del Estado, 260, 30 de octubre, 44.037-44.048. Recuperado de https://www.boe.es/eli/es/rd/2007/10/29/1393/con

REAL DECRETO 592/2014, de 11 de julio, por el que se regulan las prácticas académicas externas de los estudiantes universitarios. Boletín Oficial del Estado, 184, 30 de julio, 60.502-60.511. Recuperado de https://www.boe.es/eli/es/rd/2014/07/11/592

SUÁREZ-VILLEGAS, Juan Carlos (2015). «Aspectos éticos y deontológicos de la actividad periodística online. Su percepción por los profesionales». Revista Latina de Comunicación Social, 70, 91-109. doi: 10.4185/RLCS-2015-1036

TÚÑEZ-LÓPEZ, Miguel y MARTíNEZ-SOLANA, María Yolanda (2014). «Análisis del impacto de la función, las actitudes y las condiciones laborales del periodista en la producción de noticias: Hacia un periodismo de empresa». Zer - Revista de Estudios de Comunicación, 19(36), 169-182. Recuperado de https://www.ehu.eus/ojs/index.php/Zer/article/view/13486/12072

UNIVERSIDAD DE MÁLAGA (2016). Normativa de prácticas externas de la Universidad de Málaga. Recuperado de https://www.uma.es/secretariageneral/newsecgen/index.php?option=com_content\& view=article $\& \mathrm{id}=260$ :normativa-de-practicas-externas-de-la-universidad-de-malaga $\&$ catid $=20$ : sec-noralumnado\&Itemid $=124 \% 20$

WEAVER, David H. y WILLNAT, Lars (Eds.) (2012). The global journalist in the 21st Century: News people around the world. Nueva York, Estados Unidos: Routledge. 